\title{
Password-Based Authenticated Key Exchange in the Three-Party Setting
}

\author{
Michel Abdalla, Pierre-Alain Fouque, and David Pointcheval \\ Departement d'Informatique \\ École normale supérieure \\ 45 Rue d'Ulm, 75230 Paris Cedex 05, France \\ \{Michel.Abdalla, Pierre-Alain.Fouque, David.Pointcheval\}@ens.fr \\ http://www.di.ens.fr/users/\{mabdalla, fouque, pointche\}
}

\begin{abstract}
Password-based authenticated key exchange are protocols which are designed to be secure even when the secret key or password shared between two users is drawn from a small set of values. Due to the low entropy of passwords, such protocols are always subject to online guessing attacks. In these attacks, the adversary may succeed with non-negligible probability by guessing the password shared between two users during its on-line attempt to impersonate one of these users. The main goal of password-based authenticated key exchange protocols is to restrict the adversary to this case only. In this paper, we consider password-based authenticated key exchange in the three-party scenario, in which the users trying to establish a secret do not share a password between themselves but only with a trusted server. Towards our goal, we recall some of the existing security notions for password-based authenticated key exchange protocols and introduce new ones that are more suitable to the case of generic constructions. We then present a natural generic construction of a three-party protocol, based on any two-party authenticated key exchange protocol, and prove its security without making use of the Random Oracle model. To the best of our knowledge, the new protocol is the first provably-secure password-based protocol in the three-party setting.
\end{abstract}

Keywords: Password, authenticated key exchange, key distribution, multi-party protocols.

\section{Introduction}

Motivation. A fundamental problem in cryptography is how to communicate securely over an insecure channel, which might be controlled by an adversary. It is common in this scenario for two parties to encrypt and authenticate their messages in order to protect the privacy and authenticity of these messages. One way of doing so is by using public-key encryption and signatures, but the cost associated with these primitives may be too high for certain applications. Another way of addressing this problem is by means of a key exchange protocol, in which users establish a common key which they can then use in their applications. 
In practice, one finds several flavors of key exchange protocols, each with its own benefits and drawbacks. Among the most popular ones is the 3-party Kerberos authentication system [25]. Another one is the 2-party SIGMA protocol [17] used as the basis for the signature-based modes of the Internet Key Exchange (IKE) protocol. Yet another flavor of key exchange protocols which has received significant attention recently are those based on passwords.

Password-Based Key Exchange. Password-based key exchange protocols assume a more realistic scenario in which secret keys are not uniformly distributed over a large space, but rather chosen from a small set of possible values (a fourdigit pin, for example). They also seem more convenient since human-memorable passwords are simpler to use than, for example, having additional cryptographic devices capable of storing high-entropy secret keys. The vast majority of protocols found in practice do not account, however, for such scenario and are often subject to so-called dictionary attacks. Dictionary attacks are attacks in which an adversary tries to break the security of a scheme by a brute-force method, in which it tries all possible combinations of secret keys in a given small set of values (i.e., the dictionary). Even though these attacks are not very effective in the case of high-entropy keys, they can be very damaging when the secret key is a password since the attacker has a non-negligible chance of winning. Such attacks are usually divided in two categories: off-line and online dictionary attacks.

To address this problem, several protocols have been designed to be secure even when the secret key is a password. The goal of these protocols is to restrict the adversary's success to on-line guessing attacks only. In these attacks, the adversary must be present and interact with the system in order to be able to verify whether its guess is correct. The security in these systems usually relies on a policy of invalidating or blocking the use of a password if a certain number of failed attempts has occurred.

3-Party Password-Based Key Exchange. Passwords are mostly used because they are easier to remember by humans than secret keys with high entropy. Consequently, users prefer to remember very few passwords but not many. However, in scenarios where a user wants to communicate with many other users, then the number of passwords that he or she would need to remember would be linear in the number of possible partners. In order to limit the number of passwords that each user needs to remember, we consider in this paper password-based authenticated key exchange in the 3-party model, where each user only shares a password with a trusted server. The main advantage of this solution is that it provides each user with the capability of communicating securely with other users in the system while only requiring it to remember a single password. This seems to be a more realistic scenario in practice than the one in which users are expected to share multiple passwords, one for each party with which it may communicate privately. Its main drawback is that the server is needed during the establishment of all communication as in the Needham and Schroeder protocol.

Key PRIVACy. One potential disadvantage of a 3-party model is that the privacy of the communication with respect to the server is not always guaranteed. Since 
we want to trust as little as possible the third party, we develop a new notion called key privacy which roughly means that, even though the server's help is required to establish a session key between two users in the system, the server should not be able to gain any information on the value of that session key. Here we assume that the server is honest but curious. Please note that key distribution schemes usually do not achieve this property.

Insider ATtACKs. One of the main differences between the 2-party and the 3 -party scenarios is the existence of insider attacks. To better understand the power of these attacks, consider the protocol in Figure 1, based on the encrypted key exchange of Bellovin and Merritt[9], in which the server simply decrypts the message it receives and re-encrypts it under the other user's password. In this protocol, it is easy to see that one can mount an off-line dictionary by simply playing the role of one of the involved parties. Notice that both $A$ and $B$ can obtain the necessary information to mount an off-line dictionary attack against each other simply by eavesdropping on the messages that are sent out by the server. More specifically, $A$ and $B$ can respectively learn the values $X_{S}^{\star}=$ $\mathcal{E}_{P W_{B}}\left(X_{S}\right)$ and $Y_{S}^{\star}=\mathcal{E}_{P W_{A}}\left(Y_{S}\right)$ and mount a dictionary attack against each other using the fact that $X_{S}=X_{A}$ and $Y_{S}=Y_{B}$. Insider attacks do not need be considered explicitly in the case of 2-party protocols due to the independence among the passwords shared between pairs of honest users and those shared with malicious users.

A New Security Model. In order to analyze the security of 3-party passwordbased authenticated key exchange protocols, we put forward a new security model and define two notions of security: semantic security of the session key and key privacy with respect to the server. The first of these notions is the usual one and is a straight-forward generalization of the equivalent notion in the 2-party

Public information: $\mathbb{G}, g, p, \mathcal{E}, \mathcal{D}, H$

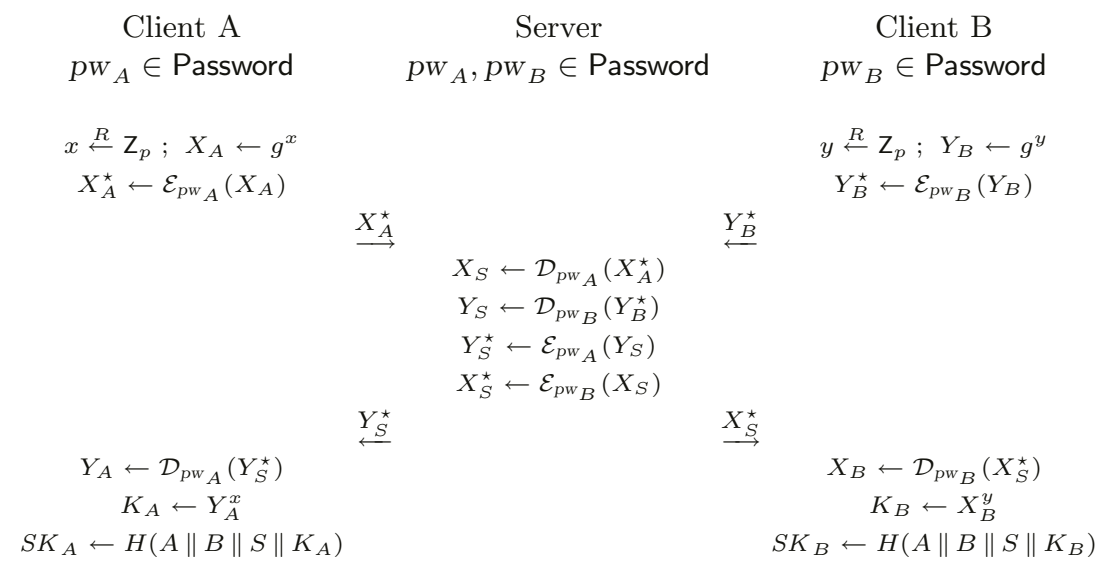

Fig. 1. An insecure 3-party password-based encrypted key exchange protocol. 
password-based authenticated key exchange model. The second one is new and particular to the new setting, and captures the privacy of the key with respect to the trusted server to which all passwords are known.

A Generic Construction. In this paper, we consider a generic construction of 3-party password-based protocol. Our construction is a natural one, building upon existing 2-party password-based key exchange and 3-party symmetric key distribution schemes, to achieve provable security in the strongest sense. Moreover, our construction is also modular in the sense that it can be broken into two parts, a 3-party password-based key distribution protocol and 2-party authenticated key exchange. The second part is only needed if key privacy with respect to the server is required.

The Need For New Security Notions. Surprisingly, the proof of security for the new scheme does not follow from the usual security notions for the underlying schemes as one would expect and requires a new and stronger notion of security for the underlying 2-party password-based scheme (see Section 2). In fact, this new security notion is not specific to password-based schemes and is one of the main contributions of this paper. Fortunately, we observe that most existing 2party password-based schemes do in fact satisfy this new property $[11,13,16$, 21]. More specifically, only a few small changes are required in their proof in order to achieve security in the new model. The bounds obtained in their proof remain essentially unchanged.

Contributions. In this paper, we consider password-based (implicitly) authenticated key exchange in the 3-party model, where each user only shares a password with a trusted server.

New Security Models. Towards our goal, we put forth a new formal security model that is appropriate for the 3-party password-based authenticated key exchange scenario and give precise definitions of what it means for it to be secure. Our model builds upon those of Bellare and Rogaway [7,8] for key distribution schemes and that of Bellare, Pointcheval, and Rogaway [5] for password-based authenticated key exchange.

New Security Notions. We also present a new and stronger model for 2party authenticated key exchange protocols, which we call the Real-Or-Random model. Our new model is provably stronger than the existing model, to which we refer to as the Find-Then-Guess model, in the sense that a scheme proven secure in the new model is also secure in the existing model. However, the reverse is not necessarily true due to an unavoidable non-constant factor loss in the reduction. Such losses in the reduction are extremely important in the case of passwordbased protocols.

A Generic Construction in the Standard Model. We present a generic and natural framework for constructing a 3-party password-based authenticated key exchange protocol from any secure 2-party password-based one. We do so by combining a 3-party key distribution scheme, an authenticated Diffie-Hellman key exchange protocol, and the 2-party password-based authenticated key exchange protocol. The proof of security relies solely on the security properties of 
underlying primitives it uses and does not assume the Random Oracle model [6]. Hence, when appropriately instantiated, this construction yields a secure protocol in the standard model.

A Separation Between Key Distribution and Key Exchange. In addition to semantic security of the session key, we present a new property, called key privacy, which is specific to key exchange protocols. This new notion captures in a quantitative way the idea that the session key shared between two instances should be only known to these two instances and no one else, including the trusted server.

Related Work. Password-based authenticated key exchange has been extensively studied in the last few years [5, 10-15, 18-20, 23, 26], with a portion of the work dealing with the subject of group key exchange and the vast majority dealing with different aspects of 2-party key exchange. Only a few of them (e.g., [12, $18,26]$ ) consider password-based protocols in the 3-party setting, but none of their schemes enjoys provable security. In fact, our generic construction seems to be the first provably-secure 3-party password-based authenticated key exchange protocol.

Another related line of research is authenticated key exchange in the 3-party setting. The first work in this area is the protocol of Needham and Schroeder [22], which inspired the Kerberos distributed system. Later, Bellare and Rogaway introduced a formal security model in this scenario along with a construction of the first provably-secure symmetric-key-based key distribution scheme [8]. In this paper, we consider the special but important case in which the secret keys are drawn from a small set of values.

Organization. In Section 2, we recall the existing security model for 2-party password-based authenticated key exchange and introduce a new one. Next, in Section 3, we introduce new models for 3-party password-based authenticated key exchange. Section 4 then presents our generic construction of a 3-party password-based authenticated key exchange protocol, called GPAKE, along with the security claims and suggestions on how to instantiate it. Some future extensions of this work are presented in Section 5. In Appendix A, we describe the cryptographic primitives and assumptions on which GPAKE is based. We conclude by presenting some results in Appendix B regarding the relation between the existing security notions and the new ones being introduced in this paper.

\section{Security Models for 2-Party Password-Based Key Exchange}

A secure 2-party password-based key exchange is a 2PAKE protocol where the parties use their password in order to derive a common session key $s k$ that will be used to build secure channels. Loosely speaking, such protocols are said to be secure against dictionary attacks if the advantage of an attacker in distinguishing a real session key from a random key is less than $O(n /|\mathcal{D}|)+\varepsilon(k)$ where $|\mathcal{D}|$ is 
the size of the dictionary $\mathcal{D}, n$ is the number of active sessions and $\varepsilon(k)$ is a negligible function depending on the security parameter $k$.

In this section, we recall the security model for 2-party password-based authenticated key exchange protocols introduced by Bellare, Pointcheval, and Rogaway (BPR) [5] and introduce a new one. For reasons that will soon become apparent, we refer to the new model as the Real-Or-Random (ROR) model and to the BPR model as the Find-Then-Guess (FTG) model, following the terminology of Bellare et al. for symmetric encryption schemes [4].

\subsection{Communication Model}

Protocol Participants. Each participant in the 2-party password-based key exchange is either a client $C \in \mathcal{C}$ or a server $S \in \mathcal{S}$. The set of all users or participants $\mathcal{U}$ is the union $\mathcal{C} \cup \mathcal{S}$.

Long-Lived Keys. Each client $C \in \mathcal{C}$ holds a password $p w_{C}$. Each server $S \in \mathcal{S}$ holds a vector $p w_{S}=\left\langle p w_{S}[C]\right\rangle_{C \in \mathcal{C}}$ with an entry for each client, where $p w_{S}[C]$ is the transformed-password, as defined in [5]. In a symmetric model, $p w_{S}[C]=p w_{C}$, but they may be different in some schemes. $p w_{C}$ and $p w_{S}$ are also called the long-lived keys of client $C$ and server $S$.

Protocol Execution. The interaction between an adversary $A$ and the protocol participants occurs only via oracle queries, which model the adversary capabilities in a real attack. During the execution, the adversary may create several concurrent instances of a participant. These queries are as follows, where $U^{i}$ denotes the instance $i$ of a participant $U$ :

- Execute $\left(C^{i}, S^{j}\right)$ : This query models passive attacks in which the attacker eavesdrops on honest executions between a client instance $C^{i}$ and a server instance $S^{j}$. The output of this query consists of the messages that were exchanged during the honest execution of the protocol.

- Send $\left(U^{i}, m\right)$ : This query models an active attack, in which the adversary may intercept a message and then either modify it, create a new one, or simply forward it to the intended participant. The output of this query is the message that the participant instance $U^{i}$ would generate upon receipt of message $m$.

\subsection{Security Definitions}

PARTNERING. We use the notion of partnering based on session identifications (sid), which says that two instances are partnered if they hold the same non-null sid. In practice, the sid is taken to be the partial transcript of the conversation between the client and the server instances before the acceptance.

FRESHNESS. In order to properly formalize security notions for the session key, one has to be careful to avoid cases in which adversary can trivially break the security of the scheme. For example, an adversary who is trying to distinguish 
the session key of an instance $U^{i}$ from a random key can trivially do so if it obtains the key for that instance through a Reveal query (see definition below) to instance $U^{i}$ or its partner. Instead of explicitly defining a notion of freshness and mandating the adversary to only perform tests on fresh instances as in previous work, we opted to embed that notion inside the definition of the oracles.

Semantic Security in the Find-Then-Guess Model. This is the definition currently being used in the literature. In order to measure the semantic security of the session key of user instance, the adversary is given access to two additional oracles: the Reveal oracle, which models the misuse of session keys by a user, and the Test oracle, which tries to capture the adversary's ability (or inability) to tell apart a real session key from a random one. Let $b$ be a bit chosen uniformly at random at the beginning of the experiment defining the semantic security in the Find-Then-Guess model. These oracles are defined as follows.

- Reveal $\left(U^{i}\right)$ : If a session key is not defined for instance $U^{i}$ or if a Test query was asked to either $U^{i}$ or to its partner, then return $\perp$. Otherwise, return the session key held by the instance $U^{i}$.

- Test $\left(U^{i}\right)$ : If no session key for instance $U^{i}$ is defined or if a Reveal query was asked to either $U^{i}$ or to its partner, then return the undefined symbol $\perp$. Otherwise, return the session key for instance $U^{i}$ if $b=1$ or a random of key of the same size if $b=0$.

The adversary in this case is allowed to ask multiple queries to the Execute, Reveal, and Send oracles, but it is restricted to ask only a single query to the Test oracle. The goal of the adversary is to guess the value of the hidden bit $b$ used by the Test oracle. The adversary is considered successful if it guesses $b$ correctly.

Let SuCC denote the event in which the adversary is successful. The ftg-akeadvantage of an adversary $\mathcal{A}$ in violating the semantic security of the protocol $P$ in the Find-Then-Guess sense and the advantage function of the protocol $P$, when passwords are drawn from a dictionary $\mathcal{D}$, are respectively

$$
\mathbf{A d v}_{P, \mathcal{D}}^{\mathrm{ftg}-\mathrm{ake}}(\mathcal{A})=2 \operatorname{Pr}[\mathrm{SUCC}]-1 \text { and } \mathbf{A} \mathbf{d} \mathbf{v}_{P, \mathcal{D}}^{\mathrm{ftg}-\mathrm{ake}}(t, R)=\max _{\mathcal{A}}\left\{\mathbf{A} \mathbf{d} \mathbf{v}_{P, \mathcal{D}}^{\mathrm{ftg}-\mathrm{ake}}(\mathcal{A})\right\}
$$

where the maximum is over all $\mathcal{A}$ with time-complexity at most $t$ and using resources at most $R$ (such as the number of queries to its oracles). The definition of time-complexity that we use henceforth is the usual one, which includes the maximum of all execution times in the experiments defining the security plus the code size [1]. Note that the advantage of an adversary that simply guesses the bit $b$ is 0 in the above definition due to the rescaling of the probabilities.

Semantic Security in the Real-Or-Random Model. This is a new definition. In the Real-Or-Random model, we only allow the adversary to ask Execute, Send, and Test queries. In other words, the Reveal oracle that exists in the FindThen-Guess model is no longer available to the adversary. Instead, we allow the adversary to ask as many Test queries as it wants to different instances. All Test queries in this case will be answered using the same value for the hidden bit $b$ that was chosen at the beginning. That is, the keys returned by the Test oracle 
are either all real or all random. However, in the random case, the same random key value should be returned for Test queries that are asked to two instances which are partnered. Please note that the Test oracle is the oracle modeling the misuse of keys by a user in this case. The goal of the adversary is still the same: to guess the value of the hidden bit $b$ used to answer Test queries. The adversary is considered successful if it guesses $b$ correctly.

Let SuCC denote the event in which the adversary is successful. The ror-akeadvantage $\operatorname{Adv}_{P, \mathcal{D}}^{\text {ror-ake }}(\mathcal{A})$ of an adversary $\mathcal{A}$ in violating the semantic security of the protocol $P$ in the Real-Or-Random sense and the advantage function $\mathbf{A d v}_{P, \mathcal{D}}^{\text {ror-ake }}(t, R)$ of the protocol $P$ are then defined as in the previous definition.

Relation Between Notions. As we prove in Appendix B, the Real-OrRandom (ROR) security model is actually stronger than the Find-Then-Guess (FTG) security model. More specifically, we show that proofs of security in the ROR model can be easily translated into proofs of security in the FTG model with only a 2 factor loss in the reduction (see Lemma 1). The reverse, however, is not necessarily true since the reduction is not security preserving. There is a loss of non-constant factor in the reduction (see Lemma 2). Moreover, the loss in the reduction cannot be avoided as there exist schemes for which we can prove such a loss in security exists (see Proposition 1).

To better understand the gap between the two notions, imagine a passwordbased scheme that was proven secure in the FTG model. By definition, the advantage of any adversary is at most $O(n /|\mathcal{D}|)+\varepsilon(k)$, where $n$ is the number of active sessions and $\varepsilon(k)$ is a negligible term. By applying the reduction, we can show that no adversary can do better than $O\left(n^{2} /|\mathcal{D}|\right)+n \cdot \varepsilon(k)$, which is not enough to guarantee the security of the same scheme in the ROR model. Note that such a gap is not as important in the case where high-entropy keys are used since both terms in the expression would be negligible.

As a consequence, we cannot take for granted the security of the existing schemes and new proofs of security need be provided. Fortunately, we would like to point out here that the security proof for several of the existing schemes can be easily modified to meet the new security goals with essentially the same bounds. The reason for that is that the security proofs of most existing password-based schemes in fact prove something stronger than what is required by the security model. More specifically, most proofs generally show that not only the session key being tested looks random, but all the keys that may be involved in a reveal query also look random to an adversary that does not know the secret password, thus satisfying the security requirements of our new model. In particular, this is the case for the KOY protocol [16] and its generalization [13], and some other schemes based on the encrypted key exchange scheme of Bellovin and Merritt [9] (e.g., $[11,21])$.

Since most existing password-based schemes do seem to achieve security in the new and stronger security model and since the latter appears to be more applicable to situations in which one wishes to use a password-based key exchange protocol as a black box, we suggest the use of our new model when proving the security of new password-based schemes. 


\section{Security Models for 3-Party Password-Based Key Exchange}

In this section, we put forward new formal security models for 3-party passwordauthenticated key exchange and key distribution protocols. Our models are generalizations of the model of Bellare and Rogaway [8] for 3-party key distribution schemes to the password case and that of Bellare, Pointcheval, and Rogaway [5] for 2-party password-based authenticated key exchange.

\subsection{Protocol Syntax}

Protocol Participants. Each participant in a 3-party password-based key exchange is either a client $U \in \mathcal{U}$ or a trusted server $S \in \mathcal{S}$. The set of clients $\mathcal{U}$ is made up of two disjoint sets: $\mathcal{C}$, the set of honest clients, and $\mathcal{E}$, the set of malicious clients. For simplicity, and without loss of generality ${ }^{1}$, we assume the set $\mathcal{S}$ to contain only a single trusted server.

The inclusion of the malicious set $\mathcal{E}$ among the participants is one the main differences between the 2-party and the 3-party models. Such inclusion is needed in the 3-party model in order to cope with the possibility of insider attacks. The set of malicious users did not need to be considered in the 2-party due to the independence among the passwords shared between pairs of honest participants and those shared with malicious users.

Long-Lived KeYs. Each participant $U \in \mathcal{U}$ holds a password $p w_{U}$. Each server $S \in \mathcal{S}$ holds a vector $\mathrm{pw}_{S}=\left\langle p w_{S}[U]\right\rangle_{U \in \mathcal{U}}$ with an entry for each client, where $p w_{S}[U]$ is the transformed-password, following the definition in [5]. In a symmetric model, $p w_{S}[U]=p w_{U}$, but they may be different in some schemes. The set of passwords $p w_{E}$, where $E \in \mathcal{E}$, is assumed to be known by the adversary.

\subsection{Communication Model}

The interaction between an adversary $A$ and the protocol participants occurs only via oracle queries, which model the adversary capabilities in a real attack. These queries are as follows:

- Execute $\left(U_{1}^{i_{1}}, S^{j}, U_{2}^{i_{2}}\right)$ : This query models passive attacks in which the attacker eavesdrops on honest executions among the client instances $U_{1}^{i_{1}}$ and $U_{2}^{i_{2}}$ and trusted server instance $S^{j}$. The output of this query consists of the messages that were exchanged during the honest execution of the protocol.

- SendClient $\left(U^{i}, m\right)$ : This query models an active attack, in which the adversary may intercept a message and then modify it, create a new one, or simply forward it to the intended client. The output of this query is the message that client instance $U^{i}$ would generate upon receipt of message $m$.

- SendServer $\left(S^{j}, m\right)$ : This query models an active attack against a server. It outputs the message that server instance $S^{j}$ would generate upon receipt of message $m$.

${ }^{1}$ This is so because we are working in the concurrent model and because all servers in the general case know all users' passwords. 


\subsection{Semantic Security}

The security definitions presented here build upon those of Bellare and Rogaway $[7,8]$ and that of Bellare, Pointcheval, and Rogaway [5].

Notation. Following $[7,8]$, an instance $U^{i}$ is said to be opened if a query Reveal $\left(U^{i}\right)$ has been made by the adversary. We say an instance $U^{i}$ is unopened if it is not opened. Similarly, we say a participant $U$ is corrupted if a query Corrupt $(U)$ has been made by the adversary. A participant $U$ is said to be uncorrupted if it is not corrupted. We say an instance $U^{i}$ has accepted if it goes into an accept mode after receiving the last expected protocol message.

PARTNERING. Our definition of partnering follows that of [5], which uses session identifications (sid). More specifically, two instances $U_{1}^{i}$ and $U_{2}^{j}$ are said to be partners if the following conditions are met: (1) Both $U_{1}^{i}$ and $U_{2}^{j}$ accept; (2) Both $U_{1}^{i}$ and $U_{2}^{j}$ share the same session identifications; (3) The partner identification for $U_{1}^{i}$ is $U_{2}^{j}$ and vice-versa; and (4) No instance other than $U_{1}^{i}$ and $U_{2}^{j}$ accepts with a partner identification equal to $U_{1}^{i}$ or $U_{2}^{j}$. In practice, as in the 2-party case, the sid could be taken to be the partial transcript before the acceptance of the conversation among all the parties involved in the protocol, a solution which may require the forwarding of messages.

Freshness. As in the 2-party case, we opted to embed the notion of freshness inside the definition of the oracles.

Semantic Security in Find-Then-Guess Model. This definition we give here is the straight-forward generalization of that of Bellare, Pointcheval, and Rogaway [5] for the 2-party case, combined with ideas of the model of Bellare and Rogaway [8] for 3-party key distribution. As in the 2-party case, we also define a Reveal oracle to model the misuse of session keys and a Test oracle to capture the adversary's ability to distinguish a real session key from a random one. Let $b$ be a bit chosen uniformly at random at the beginning of the experiment defining the semantic security in the Find-Then-Guess model. These oracles are defined as follows:

- Reveal $\left(U^{i}\right)$ : If a session key is not defined for instance $U^{i}$ or if a Test query was asked to either $U^{i}$ or to its partner, then return $\perp$. Otherwise, return the session key held by the instance $U^{i}$.

- Test $\left(U^{i}\right)$ : If no session key is defined for instance $U^{i}$ or if the intended partner of $U^{i}$ is part of the malicious set or if a Reveal query was asked to either $U^{i}$ or to its partner, then return the invalid symbol $\perp$. Otherwise, return either the session key for instance $U^{i}$ if $b=1$ or a random of key of the same size if $b=0$.

Consider an execution of the key exchange protocol $P$ by an adversary $\mathcal{A}$, in which the latter is given access to the Reveal, Execute, SendClient, SendServer, and Test oracles and asks a single Test query, and outputs a guess bit $b^{\prime}$. Such an adversary is said to win the experiment defining the semantic security if $b^{\prime}=b$, where $b$ is the hidden bit used by the Test oracle.

Let SuCC denote the event in which the adversary wins this game. The ftg-ake-advantage $\operatorname{Adv}_{P, \mathcal{D}}^{\mathrm{ftg}-\mathrm{ake}}(\mathcal{A})$ of an adversary $\mathcal{A}$ in violating the semantic 
security of the protocol $P$ in the Find-Then-Guess sense and the advantage function $\mathbf{A d v}_{P, \mathcal{D}}^{\mathrm{ftg}-\mathrm{ake}}(t, R)$ of the protocol $P$ are then defined as in previous definitions.

We say a 3-party password-based key exchange protocol $P$ is semantically secure in the Find-Then-Guess sense if the advantage $\mathbf{A} \mathbf{d} \mathbf{v}_{P \mathcal{D}}^{\mathrm{ftg}-\text { ake }}$ is only negligibly larger than $k n /|\mathcal{D}|$, where $n$ is number of active sessions and $k$ is a constant. Note that $k=1$ in the best scenario since an adversary that simply guesses the password in each of the active sessions has an advantage of $n /|\mathcal{D}|$.

Semantic Security in Real-Or-Random Model. This is a new definition. In the Real-Or-Random model, Reveal queries are no longer allowed and are replaced by Test queries. In this case, however, the adversary is allowed to ask as many Test queries as it wants.

The modifications to the Test oracle are as follows. If a Test query is asked to a client instance that has not accepted, then return the undefined $\perp$. If a Test query is asked to an instance of an honest client whose intended partner is dishonest or to an instance of a dishonest client, then return the real session key. Otherwise, the Test query returns either the real session key if $b=1$ and a random one if $b=0$, where $b$ is the hidden bit selected at random prior to the first call. However, when $b=0$, the same random key value should be returned for Test queries that are asked to two instances which are partnered. The goal of the adversary is still the same: to guess the value of the hidden bit used by the Test oracle. The adversary is considered successful if it guesses $b$ correctly.

Consider an execution of the key exchange protocol $P$ by an adversary $A$, in which the latter is given access to the Execute, SendClient, SendServer, and Test oracles, and outputs a guess bit $b^{\prime}$. Such an adversary is said to win the experiment defining the semantic security in the ROR sense if $b^{\prime}=b$, where $b$ is the hidden bit used by the Test oracle. Let SuCC denote the event in which the adversary wins this game. The ror-ake-advantage $\operatorname{Adv}_{P, \mathcal{D}}^{\text {ror-ake }}(\mathcal{A})$ of an adversary $\mathcal{A}$ in violating the semantic security of the protocol $P$ in the Real-OrRandom sense and the advantage function $\mathbf{A d v}_{P, \mathcal{D}}^{\text {ror-ake }}(t, R)$ of the protocol $P$ are then defined as in previous definitions.

\subsection{Key Privacy with Respect to the Server}

Differently from previous work, we define the notion of key privacy to capture, in a quantitative way, the idea that the session key shared between two instances should only be known to these two instances and no one else, including the trusted server. The goal of this new notion is to limit the amount of trust put into the server. That is, even though we rely on the server to help clients establish session keys between themselves, we still want to guarantee the privacy of these session keys with respect to the server. In fact, this is the main difference between a key distribution protocol (in which the session key is known to the server) and a key exchange protocol (for which the session key remains unknown to the server).

In defining the notion of key privacy, we have in mind a server which knows the passwords for all users, but that behaves in an honest but curious manner. 
For this reason, we imagine an adversary who has access to all the passwords as well as to the Execute and SendClient oracles but not to a Reveal oracle or to a SendServer oracle, since the latter can be easily simulated using the passwords. To capture the adversary's ability to tell apart the real session key shared between any two instances from a random one, we also introduce a new type of oracle, called TestPair, defined as follows, where $b$ is a bit chosen uniformly at random at the beginning of the experiment defining the notion of key privacy.

- TestPair $\left(U_{1}^{i}, U_{2}^{j}\right)$ : If client instances $U_{1}^{i}$ and $U_{2}^{j}$ do not share the same key, then return the undefined symbol $\perp$. Otherwise, return the real session key shared between client instances $U_{1}^{i}$ and $U_{2}^{j}$ if $b=1$ or a random key of the same size if $b=0$.

Consider an execution of the key exchange protocol $P$ by an adversary $A$ with access to the Execute, SendClient, and TestPair oracles and the passwords of all users, and let $b^{\prime}$ be its output. Such an adversary is said to win the experiment defining the key privacy if $b^{\prime}=b$, where $b$ is the hidden bit used by the TestPair oracle. Let SuCC denote the event in which the adversary guesses $b$ correctly. We can then define the kp-advantage $\operatorname{Adv}_{P, \mathcal{D}}^{\mathrm{kp}-\text { ake }}(\mathcal{A})$ of $\mathcal{A}$ in violating the key privacy of the key exchange protocol $P$ and the advantage function $\mathbf{A d v}_{P, \mathcal{D}}^{\mathrm{kp}-\text { ake }}(t, R)$ of $P$ as in previous definitions.

Finally, we say an adversary $\mathcal{A}$ succeeds in breaking the key privacy of a protocol $P$ if $\mathbf{A d v}_{P, \mathcal{D}}^{\mathrm{kp}-\mathrm{ake}}(\mathcal{A})$ is non-negligible.

\section{A Generic Three-Party Password-Based Protocol}

In this section, we introduce a generic construction of a 3-party password-based key exchange protocol in the scenario in which we have an honest-but-curious server. It combines a 2-party password-based key exchange, a secure key distribution protocol and a 2-party MAC-based key exchange and has several attractive features. First, it does not assume the Random Oracle (RO) model [6]. That is, if the underlying primitives do not make use of the RO model, neither does our scheme. Hence, by using schemes such as the KOY protocol [16] for the 2-party password-based key exchange and the 3-party key distribution scheme in [8], one gets a 3-part password-based protocol whose security is in the standard model. Second, if 2-party password-based key exchange protocols already exist between the server and its users in a distributed system, they can be re-used in the construction of our 3-party password-based key exchange.

Description of the Generic Solution. Our generic construction can be seen as a form of compiler transforming any secure 2-party password-based key exchange protocol $P$ into a secure password-based 3-party key exchange protocol $P^{\prime}$ in the honest-but-curious security model using a secure key distribution KD, a secure MAC scheme, and generic number-theoretic operations in a group $\mathbb{G}$ for which the DDH assumption holds (see Appendix A).

The compiler, depicted in Figure 2, works as follows. First, we use the protocol $P$ between a user $A$ and the server $S$ to establish a secure high-entropy 


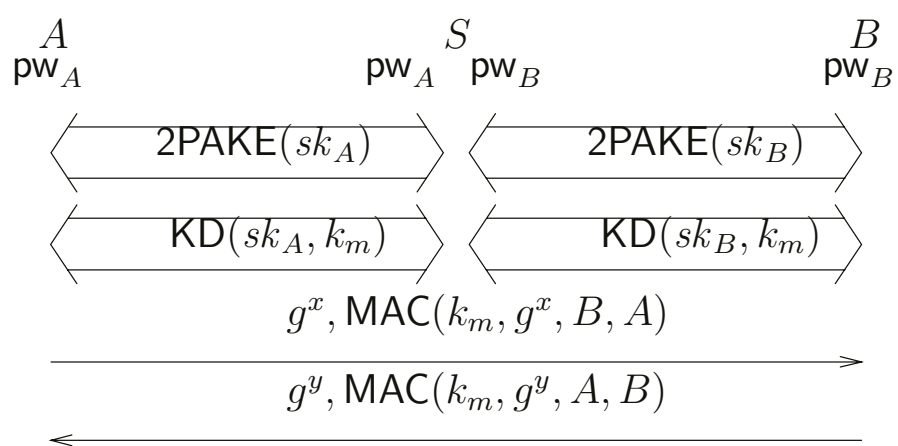

Fig. 2. GPAKE: a generic three-party password-based key exchange.

session key $s k_{A}$. Second, we use the protocol $P$ between the server $S$ and the user $B$ in order to establish a session key $s k_{B}$. Third, using a key distribution KD, we have the server $S$ first select a MAC key $k_{m}$, using the key generation of the latter, and then distribute this key to $A$ and $B$ using the session keys $s k_{A}$ and $s k_{B}$, respectively, generated in the first two steps. Finally, $A$ and $B$ use a MAC-based key exchange to establish a session key $\mathrm{CDH}$ in an authenticated way.

Semantic Security in the Real-Or-Random Model. As the following theorem states, the generic scheme GPAKE depicted in Figure 2 is a secure 3-party password-based key exchange protocol as long as the Decisional Diffie-Hellman assumption holds in $\mathbb{G}$ and the underlying primitives it uses are secure. The proof can be found in the full version of this paper [2].

Theorem 1. Let 2PAKE be a secure 2-party password-based Key Exchange, KD be a secure key distribution, and MAC be a secure MAC algorithm. Let $q_{\mathrm{exe}}$ and $q_{\text {test }}$ represent the number of queries to Execute and Test oracles, and let $q_{\mathrm{send}}^{A}, q_{\mathrm{send}}^{B}, q_{\mathrm{kd}}$, and $q_{\mathrm{ake}}$ represent the number of queries to the SendClient and SendServer oracles with respect to each of the two 2PAKE protocols, the KD protocol, and the final AKE protocol. Then,

$$
\begin{aligned}
& \mathbf{A d v}_{\mathrm{GPAKE}, \mathcal{D}}^{\mathrm{ror}-\mathrm{ake}}\left(t, q_{\mathrm{exe}}, q_{\mathrm{test}}, q_{\mathrm{send}}^{A}, q_{\mathrm{send}}^{B}, q_{\mathrm{kd}}, q_{\mathrm{ake}}\right) \leq \\
& 4 \cdot\left(q_{\mathrm{exe}}+q_{\mathrm{kd}}\right) \cdot \mathbf{A d v}_{\mathrm{KD}}^{\mathrm{ftg}-\mathrm{kd}}(t, 1,0)+2 \cdot q_{\mathrm{ake}} \cdot \mathbf{A d v}_{\mathrm{MAC}}^{\mathrm{euf}-\mathrm{cma}}(t, 2,0) \\
& +2 \cdot \mathbf{A d v}_{\mathbb{G}}^{\mathrm{ddh}}\left(t+8\left(q_{\mathrm{exe}}+q_{\mathrm{ake}}\right) \tau_{e}\right)+4 \cdot \mathbf{A d v}_{2 \mathrm{PAKE}, \mathcal{D}}^{\text {ror-ake }}\left(t, q_{\mathrm{exe}}, q_{\mathrm{exe}}+q_{\mathrm{send}}^{A}, q_{\mathrm{send}}^{A}\right) \\
& +4 \cdot \mathbf{A d v}_{2 \mathrm{PAKE}, \mathcal{D}}^{\mathrm{ror}-\text { ake }}\left(t, q_{\text {exe }}, q_{\text {exe }}+q_{\mathrm{send}}^{B}, q_{\text {send }}^{B}\right),
\end{aligned}
$$

where $\tau_{e}$ denotes the exponentiation computational time in $\mathbb{G}$.

Key Privacy with Respect to the Server. As the following theorem states, whose proof can be found in the full version of this paper [2], the generic scheme GPAKE depicted in Figure 2 has key privacy with respect to the server as long as the Decisional Diffie-Hellman assumption holds in $\mathbb{G}$.

Theorem 2. Let GPAKE be the 3-party password-based authenticated key exchange scheme depicted in Figure 2. Then, 


$$
\mathbf{A d v}_{\mathrm{GPAKE}, \mathcal{D}}^{\mathrm{kp}-\mathrm{ake}}\left(t, q_{\mathrm{exe}}, q_{\mathrm{test}}, q_{\mathrm{send}}^{A}, q_{\mathrm{send}}^{B}, q_{\mathrm{kd}}, q_{\mathrm{ake}}\right) \leq 2 \cdot \mathbf{A d v}_{\mathbb{G}}^{\mathrm{ddh}}\left(t^{\prime}\right)
$$

where $t^{\prime}=t+8 \cdot\left(q_{\text {exe }}+q_{\text {ake }}\right) \cdot \tau_{e}$ and the other parameters are defined as in Theorem 1.

Instantiations. Several practical schemes can be used in the instantiation of the 2-party password-based key exchange of our generic construction. Among them are the KOY protocol [16] and its generalization [13], the PAK suite [21], and several other schemes based on the encrypted key exchange scheme of Bellovin and Merritt [9] (e.g., [11]).

In the instantiation of the key distribution scheme, one could use the original proposal in [8] or any other secure key distribution scheme. In particular, the server could use a chosen-ciphertext secure symmetric encryption scheme to distribute the keys to the users. Independently of the choice, one should keep in mind that the security requirements for the key distribution scheme are very weak. It only needs to provide security with respect to one session.

For the instantiation of the MAC, any particular choice that makes the MAC term in Theorem 1 negligible will do. Possible choices are the HMAC [3] or the CBC MAC.

It is important to notice that, in order for GPAKE to be secure, the underlying 2-party password-based protocol must be secure in the ROR model. In view of the computational gap that exists between the ROR and the FTG models (see Proposition 1), a 2-party password-based secure in the FTG model does not suffice to prove the security of GPAKE.

\section{Concluding Remarks}

Authentication. In order to take (explicit) authentication into account, one can easily extend our model using definitions similar to those of Bellare et al. [5] for unilateral or mutual authentication. In their definition, an adversary is said to break authentication if it succeeds in making any oracle instance terminate the protocol without a partner oracle. Likewise, one could also use their generic transformation to enhance our generic construction so that it provides unilateral or mutual authentication. The drawback of using their generic transformation is that it requires the random oracle model.

More Efficient Constructions. Even though the generic construction presented in this paper is quite practical, more efficient solutions are possible. One example of such an improvement is a generic construction in which the key distribution and the final key exchange phases are combined into a single phase. One can easily think of different solutions for this scenario that are more efficient that the one we give. However, the overall gain in efficiency would not be very significant since the most expensive part of these two phases, the Diffie-Hellman protocol, seems to be necessary if key privacy with respect to the server is to be achieved. Perhaps the best way to improve the efficiency of 3-party passwordbased schemes is to adapt specific solutions in the 2-party model to the 3-party model, instead of treating these schemes as black boxes. 
Relation to Simulation Models. In [24], the Find-Then-Guess model of [8] is shown to be equivalent to simulation models in the sense that a scheme that is proven secure in one model is also secure in the other model. By closely examining their proof, one can easily see that the equivalence does not apply to the case of password-based protocols due to the non-security-preserving reduction. It seems, however, that their proof of equivalence can be adapted to show the equivalence between the simulation model and the Real-Or-Random model that we introduce in this paper in the case of password-based protocols. This is also the subject of ongoing work.

\section{Acknowledgements}

The work described in this document has been supported in part by the European Commission through the IST Programme under Contract IST-2002-507932 ECRYPT.

\section{References}

1. M. Abdalla, M. Bellare, and P. Rogaway. The oracle Diffie-Hellman assumptions and an analysis of DHIES. In CT-RSA 2001, LNCS 2020, Springer-Verlag, Apr. 2001.

2. M. Abdalla, P.-A. Fouque, and D. Pointcheval. Password-based authenticated key exchange in the three-party setting. Full version of current paper. Available from authors' web pages.

3. M. Bellare, R. Canetti, and H. Krawczyk. Keying hash functions for message authentication. In CRYPTO'96, LNCS 1109, Springer-Verlag, Aug. 1996.

4. M. Bellare, A. Desai, E. Jokipii, and P. Rogaway. A concrete security treatment of symmetric encryption. In 38th FOCS, Oct. 1997.

5. M. Bellare, D. Pointcheval, and P. Rogaway. Authenticated key exchange secure against dictionary attacks. In EUROCRYPT 2000, LNCS 1807, Springer-Verlag, May 2000.

6. M. Bellare and P. Rogaway. Random oracles are practical: A paradigm for designing efficient protocols. In ACM CCS 93, Nov. 1993.

7. M. Bellare and P. Rogaway. Entity authentication and key distribution. In CRYPTO'93, LNCS 773, Springer-Verlag, Aug. 1994.

8. M. Bellare and P. Rogaway. Provably secure session key distribution - the three party case. In 28th ACM STOC, May 1996.

9. S. M. Bellovin and M. Merritt. Encrypted key exchange: Password-based protocols secure against dictionary attacks. In 1992 IEEE Symposium on Security and Privacy, May 1992.

10. V. Boyko, P. MacKenzie, and S. Patel. Provably secure password-authenticated key exchange using Diffie-Hellman. In EUROCRYPT 2000, LNCS 1807, SpringerVerlag, May 2000.

11. E. Bresson, O. Chevassut, and D. Pointcheval. New security results on encrypted key exchange. In PKC 2004, LNCS 2947, Springer-Verlag, Mar. 2004.

12. J. W. Byun, I. R. Jeong, D. H. Lee, and C.-S. Park. Password-authenticated key exchange between clients with different passwords. In ICICS 02, LNCS 2513 , Springer-Verlag, Dec. 2002.

13. R. Gennaro and Y. Lindell. A framework for password-based authenticated key exchange. In EUROCRYPT 2003, LNCS 2656, Springer-Verlag, May 2003. 
14. O. Goldreich and Y. Lindell. Session-key generation using human passwords only. In CRYPTO 2001, LNCS 2139, Springer-Verlag, Aug. 2001.

15. S. Halevi and H. Krawczyk. Public-key cryptography and password protocols. In ACM Transactions on Information and System Security, pages 524-543. ACM, 1999.

16. J. Katz, R. Ostrovsky, and M. Yung. Efficient password-authenticated key exchange using human-memorable passwords. In EUROCRYPT 2001, LNCS 2045, Springer-Verlag, May 2001.

17. H. Krawczyk. SIGMA: The "SIGn-and-MAc" approach to authenticated DiffieHellman and its use in the ike protocols. In CRYPTO 2003, LNCS 2729, SpringerVerlag, Aug. 2003.

18. C.-L. Lin, H.-M. Sun, and T. Hwang. Three-party encrypted key exchange: Attacks and a solution. ACM SIGOPS Operating Systems Review, 34(4):12-20, Oct. 2000.

19. P. MacKenzie, S. Patel, and R. Swaminathan. Password-authenticated key exchange based on RSA. In ASIACRYPT 2000, LNCS 1976, Springer-Verlag, Dec. 2000 .

20. P. MacKenzie, T. Shrimpton, and M. Jakobsson. Threshold password-authenticated key exchange. In CRYPTO 2002, LNCS 2442, Springer-Verlag, Aug. 2002.

21. P. D. MacKenzie. The PAK suite: Protocols for password-authenticated key exchange. Contributions to IEEE P1363.2, 2002.

22. R. Needham and M. Schroeder. Using encryption for authentication in large networks of computers. Communications of the ACM, 21(21):993-999, Dec. 1978.

23. M. D. Raimondo and R. Gennaro. Provably secure threshold password-authenticated key exchange. In EUROCRYPT 2003, LNCS 2656, Springer-Verlag, May 2003.

24. V. Shoup. On formal models for secure key exchange. Technical Report RZ 3120, IBM, 1999.

25. J. G. Steiner, B. C. Neuman, and J. L. Schiller. Kerberos: An authentication service for open networks. In Proceedings of the USENIX Winter Conference, pages 191-202, 1988.

26. M. Steiner, G. Tsudik, and M. Waidner. Refinement and extension of encrypted key exchange. ACM SIGOPS Operating Systems Review, 29(3):22-30, July 1995.

\section{A Building Blocks}

Decisional Diffie-Hellman Assumption: DDH. The DDH assumption states, roughly, that the distributions $\left(g^{u}, g^{v}, g^{u v}\right)$ and $\left(g^{u}, g^{v}, g^{w}\right)$ are computationally indistinguishable when $u, v, w$ are drawn at random from $\{1, \ldots,|\mathbb{G}|\}$. This can be made more precise by defining two experiments, $\operatorname{Exp}_{\mathbb{G}}^{\text {ddh-real }}(\mathcal{A})$ and $\operatorname{Exp}_{\mathbb{G}}^{\text {ddh-rand }}(\mathcal{A})$. In both experiments, we compute two values $U=g^{u}$ and $V=g^{v}$ to be given to $\mathcal{A}$. But in addition to that, we also provide a third input, which is $g^{u v}$ in $\operatorname{Exp}_{\mathbb{G}}^{\text {ddh-real }}(\mathcal{A})$ and $g^{z}$ for a random $z$ in $\operatorname{Exp}_{\mathbb{G}}^{\text {ddh-rand }}(\mathcal{A})$. The goal of the adversary is to guess a bit indicating the experiment it thinks it is in. We define the advantage of $\mathcal{A}$ in violating the DDH assumption, $\operatorname{Adv}_{\mathbb{G}}^{\mathrm{ddh}}(\mathcal{A})$, as $\operatorname{Pr}\left[\operatorname{Exp}_{\mathbb{G}}^{\mathrm{ddh}-\mathrm{real}}(\mathcal{A})=1\right]-\operatorname{Pr}\left[\operatorname{Exp}_{\mathbb{G}}^{\mathrm{ddh}-\mathrm{rand}}(\mathcal{A})=1\right]$. The advantage function of the group, $\mathbf{A} \mathbf{d} \mathbf{v}_{\mathbb{G}}^{\mathrm{ddh}}(t)$ is then defined as the maximum value of $\mathbf{A d v}_{\mathbb{G}}^{\mathrm{ddh}}(\mathcal{A})$ over all $\mathcal{A}$ with time-complexity at most $t$. 
Message Authentication Codes (MAC). A Message Authentication Code $\mathrm{MAC}=($ Key, Tag, Ver $)$ is defined by the following three algorithms: (1) A MAC key generation algorithm Key, which on input $1^{k}$, produces a $\ell$-bit secret-key $s k$ uniformly distributed in $\{0,1\}^{\ell}$; (2) A MAC generation algorithm Tag, possibly probabilistic, which given a message $m$ and a secret key $s k \in\{0,1\}^{\ell}$, produces an authenticator $\mu$; and (3) A MAC verification algorithm Ver, which given an authenticator $\mu$, a message $m$, and a secret key $s k$, outputs 1 if $\mu$ is a valid authenticator for $m$ under $s k$ and 0 otherwise.

Like in signature schemes, the classical security level for a MAC is to prevent existential forgeries, even for an adversary which has access to the generation and verification oracles. We define the advantage of $\mathcal{A}, \operatorname{Adv}_{\mathrm{MAC}}^{\text {euf }-\mathrm{cma}}(\mathcal{A})$, as

$$
\operatorname{Pr}\left[s k \leftarrow\{0,1\}^{\ell},(m, \mu) \leftarrow \mathcal{A}^{\operatorname{Tag}(s k ; \cdot), \operatorname{Ver}(s k ; \cdot, \cdot)}(): \operatorname{Ver}(s k ; m, \mu)=1\right],
$$

and the advantage function of the MAC, $\mathbf{A d v}_{\mathrm{MAC}}^{\text {euf-cma }}\left(t, q_{g}, q_{s}\right)$, as the maximum value of $\mathbf{A} \mathbf{d} \mathbf{v}_{\mathrm{MAC}}^{\text {euf }}-\mathrm{cma}(\mathcal{A})$ over all $\mathcal{A}$ that asks up to $q_{g}$ and $q_{v}$ queries to the generation and verification oracles, respectively, and with time-complexity at most $t$. Note that $\mathcal{A}$ wins the above experiment only if it outputs a new valid authenticator.

3-Party Key Distribution. A secure key distribution protocol KD is a 3-party protocol between 2 parties and a trusted server $S$ where $S$ picks a session key at random and securely sends it to the users. The security model, formally introduced in [8], is a generalization of that for 2-party authenticated key exchange protocols, to which a new oracle was added to represent the trusted server. Their security is in the Find-Then-Guess model, using the terminology that we introduced for key exchange protocols.

In our generic construction, we only need a KD secure with respect to a single session since the symmetric keys used as input to the key distribution protocol differ from session to session. They are the session keys obtained from the 2-party password-based authenticated key exchange protocols between the server and each of the two parties. Since in this case, both the Find-Then-Guess and Real-Or-Random notions are equivalent, we opted to use their definition (i.e. FTG) adapted to our terminology. That is, we define $\operatorname{Adv}_{\mathrm{KD}}^{\mathrm{ftg}-\mathrm{kd}}(\mathcal{A})$ as the advantage of adversary $\mathcal{A}$ in violating the semantic security of a key distribution $\mathrm{KD}$ in the FTG sense, and $\mathbf{A} \mathbf{d} \mathbf{v}_{\mathrm{KD}}^{\mathrm{ftg}-\mathrm{kd}}(t, s, r)$ as the advantage function of $\mathrm{KD}$, which is the maximum value of $\operatorname{Adv}_{\mathrm{KD}}^{\mathrm{ftg}-\mathrm{kd}}(\mathcal{A})$ over all $\mathcal{A}$ with time-complexity at most $t$, asking Send queries with respect to at most $s$ sessions and asking at most $r$ Reveal queries.

\section{B Relations Between Notions}

In this section, we prove the relation between the Find-Then-Guess (FTG) and Real-Or-Random (ROR) notions of security for authenticated key exchange protocols. The relation is not specific to password-based schemes, but its implications are more important in that scenario. We do not present proofs for the 
forward-secure case as these proofs can be easily derived from the proofs in the non-forward-secure case.

Lemma 1. For any AKE, $\mathbf{A d v}_{\mathrm{AKE}}^{\mathrm{ftg}-\mathrm{ake}}\left(t, q_{\text {send }}, q_{\text {reveal }}, q_{\mathrm{exe}}\right) \leq 2 \cdot \mathbf{A d v}_{\mathrm{AKE}}^{\mathrm{ror}-\mathrm{ake}}(t$, $\left.q_{\text {send }}, q_{\text {reveal }}+1, q_{\text {exe }}\right)$.

Proof. In order to prove this lemma, we show how to build an adversary $\mathcal{A}_{\text {ror }}$ against the semantic security of an authenticated key exchange AKE protocol in the ROR model given an adversary $\mathcal{A}_{\text {ftg }}$ against the semantic security of the same protocol AKE in the FTG model. We know that $\mathcal{A}_{\mathrm{ftg}}$ has time-complexity at most $t$ and that it asks at most $q_{\text {send }}, q_{\text {reveal }}$, and $q_{\text {exe }}$ queries to its Send, Reveal, and Execute oracles, respectively.

The description of $\mathcal{A}_{\text {ror }}$ is as follows. $\mathcal{A}_{\text {ror }}$ starts by choosing a bit $b$ uniformly at random and starts running $\mathcal{A}_{\text {ftg }}$. If $\mathcal{A}_{\text {ftg }}$ asks a Send query, then $\mathcal{A}_{\text {ror }}$ asks the corresponding query to its Send oracle. If $\mathcal{A}_{\mathrm{ftg}}$ asks a Execute query, then $\mathcal{A}_{\text {ror }}$ asks the corresponding query to its Execute oracle. If $\mathcal{A}_{\mathrm{ftg}}$ asks a Reveal query, then $\mathcal{A}_{\text {ror }}$ asks a Test query to its Test oracle and uses the answer it receives as the answer to the Reveal query. If $\mathcal{A}_{\text {ftg }}$ asks a Test query, then $\mathcal{A}_{\text {ror }}$ asks the corresponding query to its Test oracle. If $b=1$, then $\mathcal{A}_{\text {ror }}$ uses the answer it received as the answer to the Test query. Otherwise, it returns a random key to $\mathcal{A}_{\mathrm{ftg}}$. Let $b^{\prime}$ be the final output of $\mathcal{A}_{\mathrm{ftg}}$. If $b^{\prime}=b$, then $\mathcal{A}_{\text {ror }}$ outputs 1 . Otherwise, it outputs 0 .

Note that $\mathcal{A}_{\text {ror }}$ has time-complexity at most $t$ and asks at most $q_{\text {send }}, q_{\text {reveal }}+$ 1 , and $q_{\text {exe }}$ queries to its Send, Test, and Execute oracles, respectively.

In order to analyze the advantage of $\mathcal{A}_{\text {ror }}$, first consider the case in which its Test oracle returns random keys. It is easy to see that, in this case, $\mathcal{A}_{\mathrm{ftg}}$ cannot gain any information about the hidden bit $b$ used to answer its single Test query. Therefore, the probability that $\mathcal{A}_{\text {ror }}$ outputs 1 is exactly $\frac{1}{2}$. Now consider the case in which its Test oracle returns the actual sessions keys. In this case, the simulation of Reveal is perfect and $\mathcal{A}_{\text {ror }}$ runs $\mathcal{A}_{\mathrm{ftg}}$ exactly as in the experiment defining the semantic security of $\mathcal{A}_{\mathrm{ftg}}$ in the FTG model. Therefore, the probability that $\mathcal{A}_{\text {ror }}$ outputs 1 is exactly $\frac{1}{2}+\frac{1}{2} \mathbf{A} \mathbf{d} \mathbf{v}_{\mathrm{AKE}}^{\mathrm{ftg}-\text { ake }}\left(\mathcal{A}_{\mathrm{ftg}}\right)$ and, as a result, $\mathbf{A d v}_{\text {AKE }}^{\mathrm{ftg}-\text { ake }}\left(\mathcal{A}_{\mathrm{ftg}}\right) \leq 2 \cdot \mathbf{A} \mathbf{d v}_{\text {AKE }}^{\text {ror-ake }}\left(\mathcal{A}_{\text {ror }}\right) \leq \mathbf{A d v}_{\text {AKE }}^{\text {ror-ake }}\left(t, q_{\text {send }}, q_{\text {reveal }}+\right.$ $\left.1, q_{\text {exe }}\right)$. The lemma follows easily.

Lemma 2. For any AKE, $\mathbf{A d v}_{\text {AKE }}^{\text {ror-ake }}\left(t, q_{\text {send }}, q_{\text {test }}, q_{\text {exe }}\right) \leq q_{\text {test }} \cdot \mathbf{A d v}_{\mathrm{AKE}}^{\mathrm{ftg}-\text { ake }}(t$, $\left.q_{\text {send }}, q_{\text {test }}-1, q_{\text {exe }}\right)$.

Proof. In order to prove this lemma, we show how to build a sequence of adversaries $\mathcal{A}_{\mathrm{ftg}}^{i}$ against the semantic security of an authenticated key exchange AKE protocol in the FTG model given an adversary $\mathcal{A}_{\text {ror }}$ against the semantic security of the same protocol AKE in the ROR model. We know that $\mathcal{A}_{\text {ror }}$ has time-complexity at most $t$ and that it asks at most $q_{\text {send }}, q_{\text {test }}$, and $q_{\text {exe }}$ queries to its Send, Test, and Execute oracles, respectively.

The proof uses a standard hybrid argument, in which we define a sequence of $q_{\text {test }}+1$ hybrid experiments $V_{i}$, where $0 \leq i \leq q_{\text {test }}$. In experiment $V_{i}$, the first $i-1$ queries to the Test oracle are answered using a random key and all remaining Test 
queries are answered using the real key. Please note that the hybrid experiments at the extremes correspond to the real and random experiments in the definition of semantic security in the ROR model. Hence, in order to prove the bound in the lemma, it suffices to prove that the difference in probability that adversary $\mathcal{A}_{\text {ror }}$ returns 1 between any two consecutive experiments $V_{i}$ and $V_{i-1}$ is at most $\mathbf{A d v}_{\text {AKE }}^{\mathrm{ftg}-\mathrm{ake}}\left(t, q_{\text {send }}, q_{\text {test }}-1, q_{\text {exe }}\right)$. This is achieved by building a sequence of $q_{\text {test }}$ adversaries $\mathcal{A}_{\mathrm{ftg}}^{i}$, as described below.

Let $\mathcal{A}_{\mathrm{ftg}}^{i}$ be a distinguisher $\mathcal{A}_{\mathrm{ftg}}^{i}$ for experiments $V_{i}$ and $V_{i-1}$, where $1 \leq i \leq$ $q_{\text {test }} \cdot \mathcal{A}_{\text {ftg }}^{i}$ starts running $\mathcal{A}_{\text {ror }}$ answering to its queries as follows. If $\mathcal{A}_{\text {ror }}$ asks a Send or Execute query, then $\mathcal{A}_{\mathrm{ftg}}$ answers it using its corresponding oracle. If $\mathcal{A}_{\text {ror }}$ asks a Test query, then $\mathcal{A}_{\text {ftg }}$ answers it with a random key if this query is among the first $i-1$. If this is the $i$-th Test, then $\mathcal{A}_{\mathrm{ftg}}$ uses its Test oracle to answer it. All remaining Test queries are answered using the output of the Reveal query. $\mathcal{A}_{\text {ftg }}$ finishes its execution by outputting the same guess bit $b$ outputted by $\mathcal{A}_{\text {ror }}$.

Note that $\mathcal{A}_{\text {ftg }}^{i}$ has time-complexity at most $t$ and asks at most $q_{\text {send }}, q_{\text {test }}-1$, and $q_{\text {exe }}$ queries to its Send, Reveal, and Execute oracles, respectively.

In order to analyze the advantage of $\mathcal{A}_{\mathrm{ftg}}^{i}$, first notice that when Test oracle returns a random key, $\mathcal{A}_{\mathrm{ftg}}^{i}$ runs $\mathcal{A}_{\text {ror }}$ exactly as in the experiment $V_{i}$. Next, notice that when Test oracle returns the real key, $\mathcal{A}_{\mathrm{ftg}}^{i}$ runs $\mathcal{A}_{\text {ror }}$ exactly as in the experiment $V_{i}$. It follows that the difference in probability that adversary $\mathcal{A}_{\text {ror }}$ returns 1 between experiments $V_{i}$ and $V_{i-1}$ is at most $\mathbf{A d v}_{\mathrm{AKE}}^{\mathrm{ftg}-\mathrm{ake}}\left(\mathcal{A}_{\text {ror }}\right) \leq$ $\mathbf{A d v}_{\text {AKE }}^{\mathrm{ftg}-\mathrm{ake}}\left(t, q_{\text {send }}, q_{\text {test }}-1, q_{\text {exe }}\right)$. The lemma follows easily.

Even though the reduction in Lemma 2 is not security-preserving (i.e., there is a non-constant factor loss in the reduction), it does not imply that a gap really exists - there might exist a tight reduction between the two notions that we have not yet found. In order to prove that the non-constant factor loss in the reduction is indeed intrinsic, we need to show that there exist schemes for which the gap does exist.

To achieve this goal, one can use techniques similar to those used to prove that a gap exists between the Left-Or-Right and Find-Then-Guess notions of security for symmetric encryption schemes [4]. In that paper, they show how to construct a new symmetric encryption scheme $\mathcal{E}^{\prime}$ from a secure encryption scheme $\mathcal{E}$ such that $\mathcal{E}^{\prime}$ exhibits the gap. $\mathcal{E}^{\prime}$ was constructed in such a way that its encryption function works like the encryption function of $\mathcal{E}$ most of the time, except in a few cases (which are easily identifiable) in which the ciphertext it generates contains the plaintext. The probability in which such bad cases happen in their construction is exactly $1 / q$, where $q$ is the non-constant factor in the reduction.

A similar technique can be applied to authenticated key exchange protocols. Imagine a secure authenticated key exchange protocol AKE exists. For simplicity, assume $q_{\text {test }}=2^{l}$, for some integer $l$. We can construct a new scheme AKE' such that the session key $k$ that it generates equals the one generated by AKE most of the time except when the first $l$ bits are 0 . In this case, we just make $k=0$. Using a proof technique similar to that used in [4], one can prove the the gap 
in Lemma 2 cannot be avoided and we thus omit the detail. But before stating our proposition, we make a final remark that when the underlying scheme AKE is a password-based key exchange, not every choice of parameters will yield the desired result claimed in the proposition. However, there are (easy) choices of parameters for which the gap does exist and that suffices for the purpose of the proposition. We are now ready to state our claim.

Proposition 1. The gap exhibited in Lemma 2 is intrinsic and cannot be avoided. 